\title{
Stability of Uncertain Piecewise Affine Systems with Time-Delay: Delay-Dependent Lyapunov Approach
}

\author{
KAVEH MOEZZI $\dagger$, LUIS RODRIGUESłand AMIR G. AGHDAM $\dagger \S$ \\ $\dagger$ Department of Electrical and Computer Engineering, Concordia University Montréal, QC Canada H3G 1M8, CA \\ ‡ Department of Mechanical and Industrial Engineering, Concordia University, Montréal, QC, H3G 1M8, CA \\ (Received 00 Month 200x; in final form 00 Month 200x)
}

This paper addresses the problem of robust stability of piecewise affine (PWA) uncertain systems with unknown time-varying delay in the state. It is assumed that the uncertainty is norm-bounded and that the upper bounds on the state delay and its rate of change are available. A set of LMIs is derived providing sufficient conditions for the stability of the system. These conditions depend on the upper bound of the delay. The main contributions of the paper are as follows. First, new delay-dependent linear matrix inequality (LMI) conditions are derived for the stability of PWA time-delay systems. Second, the stability conditions are extended to the case of uncertain PWA time-delay systems. Numerical examples are presented to show the effectiveness of the approach.

\section{Introduction}

Continuous-time piecewise affine (PWA) systems have attracted considerable interest in the control literature in recent years (Johansson 2003), (Kulkarni et al. 2004), (Rodrigues 2004), (Hassibi and Boyd 1998), (Rodrigues and Boyd 2005), (Rodrigues and How 2006), (Rodrigues and Boukas 2006), (Rodrigues and How 2003). The theory of PWA systems has found important applications in CPU processing control (Azuma and Imura 2003), boost DC-DC converters (Beccuti et al. 2005) and aerospace (Wei et al. 2006), to name only a few. In brief, a PWA system consists of a set of affine subsystems (representing the different operating conditions of a system, or an approximation of a complex nonlinear system) and a switching law that enables switching between different subsystems. It is to be noted that switching is also used in control to stabilize and regulate highly uncertain systems (Momeni and Aghdam 2007), (Aghdam and Davison 2003), (Aghdam and Davison 2007), (Tousi et al. 2008).

Many practical systems are subject to input and/or state delay. Examples of time-delay systems include power systems (Bibian and Jin 2000) and communication networks (Zhang et al. 2001). It is known that time-delay can cause poor performance or even instability if its effect is neglected in control design. The existing results for robust stability of time-delay systems can be categorized as delay independent and delay dependent results. Different delay independent robust stability criteria have been developed in (Verriest et al. 1993) and (Wang et al. 1987). Delay independent stability results are conservative, in general because they do not take into account any available information on the delay. Delay dependent approaches for the systems subject to parameter uncertainty, on the other hand, are investigated in (Fridman and Shaked 2003), (C. Lin and Lee 2006), (Su 1994), (Han and Gu 2001), (Parlakci 2006). Stability analysis for switched systems with time-delay is provided in (Sun et al. 2006b), (Zhai et al. 2003), (Sun et al. 2006a). In (Sun et al. 2006b), a common Lyapunov functional is used for robust stability analysis of switched uncertain time-delay systems with arbitrary switching. However, any stability analysis using a common quadratic Lyapunov function is typically known to be conservative. In (Sun et al. 2006a), sufficient conditions for exponential stability of linear time-delay systems with a class of switching signals is developed. To the best of the knowledge of the authors, however, the stability problem for PWA time-delay systems has only been addressed in (Kulkarni et al. 2004), where a piecewise quadratic Lyapunov function is used to derive LMIs for stability analysis following the approach of (Johansson 2003). Nevertheless, the important

$\S$ Author for Correspondence: email: aghdam@ece.concordia.ca 
and practically relevant case of robust stability of PWA time-delay systems in presence of parametric uncertainty has not been considered in (Kulkarni et al. 2004). Furthermore, the affine term of the dynamics did not have a delay in that paper.

Based on the considerations of the previous paragraph, PWA uncertain systems with unknown time-delay are investigated in this paper, and LMI-based conditions for asymptotic stability are derived following the approach of (Rodrigues and How 2003). It is assumed that the parameter uncertainties are norm bounded and that upper bounds on the time-varying delay and its rate of change are given. In order to reduce the conservativeness of the results, piecewise quadratic Lyapunov functions are employed for stability analysis. The main contributions of this work are as follows. First, new delay dependent LMI conditions are derived for the stability of PWA time-delay systems. Second, the stability conditions are extended to the case of uncertain PWA time-delay systems.

This paper is organized as follows. The problem statement and formulation are given in Section 2. The main result of the paper is provided in Section 3, followed by robustness analysis in Section 4. Simulation results are presented in Section 5. Finally, some concluding remarks are drawn in Section 6.

\section{Problem Formulation}

Consider an uncertain piecewise affine system with time-delay described as

$$
\dot{x}(t)=\left(A_{i}+\Delta A_{i}\right) x(t)+\left(A_{d i}+\Delta A_{d i}\right) x(t-\tau(t))+\left(a_{i}+\Delta a_{i}\right)+\left(b_{i}+\Delta b_{i}\right) 1(t-\tau(t)), \quad x(t) \in X_{i}
$$

where $A_{i}, A_{d i} \in R^{n \times n}, a_{i}, b_{i} \in R^{n}$, and $\left\{X_{i}\right\} \subseteq R^{n}$ form a partition of the state space into a number of open (possibly unbounded) polyhedral cells with pairwise empty intersection. The index set of the cells is denoted by $I=\{1, \ldots, M\}$. The set of cells that include the origin is denoted by $I_{0} \subseteq I$, and its complement is represented by $I_{1}=I / I_{0}$. It is assumed that $a_{i}=0, \Delta a_{i}=0, b_{i}=0, \Delta b_{i}=0$ for $i \in I_{0}$. In addition, $\Delta A_{i}, \Delta A_{d i}, \Delta a_{i}$ and $\Delta b_{i}$ are norm-bounded uncertainties which will be defined later. Furthermore, $1(t)$ is the step function. In $(1), \tau(t)$ is a positive time-varying delay such that

$$
0 \leq \tau(t) \leq h, \quad \dot{\tau}(t) \leq d<1
$$

where $h$ and $d$ are positive constants.

Assume the initial condition

$$
x(\theta)=\phi(\theta), \quad \theta \in[-h, 0]
$$

for the system (1) such that $\phi(\theta)$ is a differentiable vector-valued initial function on $[-h, 0], h>0$. Assume also $x(t)$ is a continuous piecewise $C^{1}$ function of time. Following (Johansson 2003), (Rodrigues and How 2003), the state space is partitioned based on $x(t)$ such that $x(t) \in \bigcup X_{i}$ as follows. Let $\bar{E}_{i}=\left[E_{i} e_{i}\right]$, $\left(e_{i}=0, \forall i \in I_{0}\right)$, such that

$$
\bar{E}_{i}\left[\begin{array}{c}
x(t) \\
1
\end{array}\right] \geq 0 \quad \forall x(t) \in X_{i}, i \in I
$$

Let $\mathcal{N}_{i}$ denote the set of neighbouring cells that share a common facet with the cell $X_{i}$. The facet boundary between the cells $X_{i}$ and $X_{k}$ is contained in the set $\left\{x \in R^{n} \mid c_{i k}^{T} x(t)-d_{i k}=0\right\}$, where $c_{i k} \in R^{n}, d_{i k} \in R$, for all $i \in I, k \in \mathcal{N}_{i}$. Moreover, assume the description of the boundaries as follows

$$
\bar{X}_{i} \cap \bar{X}_{k} \subseteq\left\{l_{i k}+F_{i k} s \mid s \in R^{n-1}\right\}
$$

for all $i \in I, k \in \mathcal{N}_{i}$, where $F_{i k} \in R^{n \times(n-1)}$ is a full rank matrix whose columns span the null space of $c_{i k}^{T}$ and $l_{i k} \in R^{n}$ is given by $l_{i k}=c_{i k}\left(c_{i k}^{T} c_{i k}\right)^{-1} d_{i k}$. 
The main objective of this paper is to determine a set of computationally tractable conditions under which (1) is asymptotically stable. In the next section, a Lyapunov functional will be introduced to determine the stability of PWA systems.

\section{Nominal Analysis}

In this section, sufficient LMI conditions will be established for the stability of (1) without uncertainties. These conditions will be extended to the systems with uncertainties in Section 4. To proceed further, we define the following matrices and sets

$$
\begin{aligned}
& \bar{A}_{i}:=\left[\begin{array}{cc}
A_{i} & a_{i} \\
0 & 0
\end{array}\right], \quad \bar{A}_{d i}:=\left[\begin{array}{cc}
A_{d i} & 0 \\
0 & 0
\end{array}\right], \quad \bar{b}_{i}:=\left[\begin{array}{c}
b_{i} \\
0
\end{array}\right] \\
& \overline{\mathcal{A}}=\left\{\left[\begin{array}{cc}
A_{j} & a_{j} \\
0 & 0
\end{array}\right], \forall j \in I\right\}, \quad \mathcal{A}=\left\{A_{j}, \forall j \in I\right\}, \quad \mathcal{E}=\left\{a_{j}, \forall j \in I_{1}\right\} \\
& \overline{\mathcal{B}}=\left\{\left[\begin{array}{c}
b_{j} \\
0
\end{array}\right], \forall j \in I_{1}\right\}, \quad \mathcal{B}=\left\{b_{j}, \forall j \in I_{1}\right\} \\
& \overline{\mathcal{A}}_{d}=\left\{\left[\begin{array}{cc}
A_{d j} & 0 \\
0 & 0
\end{array}\right], \forall j \in I\right\}, \quad \mathcal{A}_{d}=\left\{A_{d j}, \forall j \in I\right\}
\end{aligned}
$$

Note that system (1) without uncertainties can be rewritten as follows

$$
\dot{\bar{x}}=\bar{A}_{i} \bar{x}(t)+\bar{A}_{d i} \bar{x}(t-\tau(t))+\bar{b}_{i} 1(t-\tau(t))
$$

where $\bar{x}(t)=\left[x^{T}(t), 1\right]^{T}$ and $\bar{x}(t-\tau(t))=\left[x^{T}(t-\tau(t)), 1\right]^{T}$, with $x(t) \in X_{i}$. We use the expression

$$
\bar{x}(t-\tau(t))=\bar{x}(t)-\int_{t-\tau(t)}^{t} \dot{\bar{x}}(s) d s
$$

Hence, considering (5), the equation (6) can be rewritten as

$$
\begin{aligned}
\dot{\bar{x}}(t)= & \left(\bar{A}_{i}+\bar{A}_{d i}\right) \bar{x}(t)+\bar{b}_{i} 1(t-\tau(t))-\bar{A}_{d i} \int_{t-\tau(t)}^{t} \bar{A}_{j(s)} \bar{x}(s) d s \\
& -\bar{A}_{d i} \int_{t-\tau(t)}^{t} \bar{A}_{d j(s)} \bar{x}(s-\tau(s)) d s-\bar{A}_{d i} \int_{t-\tau(t)}^{t} \bar{b}_{j(s)} 1(s-\tau(s)) d s
\end{aligned}
$$

Note that $j(s)$ in (7) is a piecewise constant function which represents the index of the matrices $\bar{A}_{j(s)} \in \overline{\mathcal{A}}$, $\bar{b}_{j(s)} \in \overline{\mathcal{B}}, \bar{A}_{d j(s)} \in \overline{\mathcal{A}}_{d}$ at time $s$. In order to proceed further, the following well-known lemma is borrowed from (Wang et al. 1992).

Lemma 1: For any vectors or matrices $z$ and $y$ with appropriate dimensions and any symmetric matrix 
$P>0$, the following inequalities are satisfied:

$$
\begin{gathered}
-z^{T} y-y^{T} z \leq z^{T} P z+y^{T} P^{-1} y \\
z^{T} y+y^{T} z \leq z^{T} P z+y^{T} P^{-1} y
\end{gathered}
$$

Proof: See (Wang et al. 1992).

The following Theorem presents sufficient conditions for the stability of the PWA system (5).

Theorem 1: Consider symmetric matrices $\bar{U}_{i}, U_{i}$ and $\bar{W}_{i}, W_{i}$, where $\bar{U}_{i}, U_{i}$ and $\bar{W}_{i}, W_{i}$ are composed of non-negative entries, and

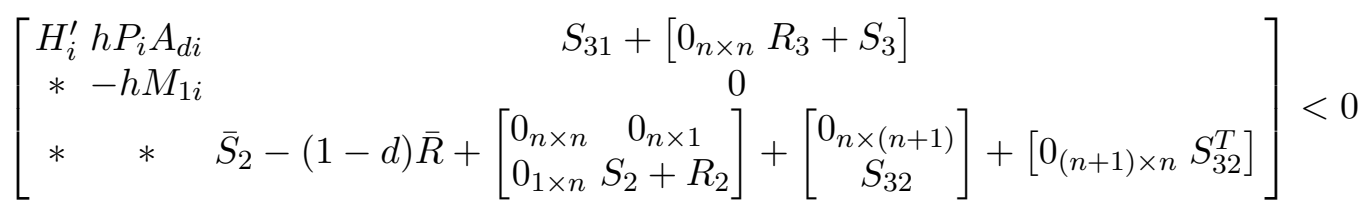

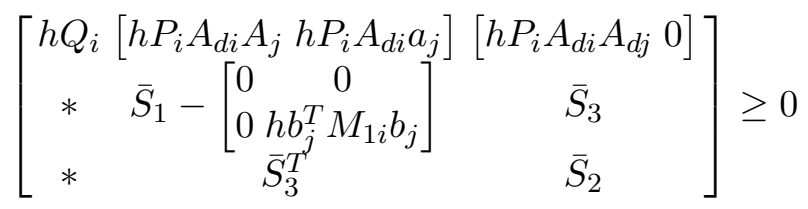

$$
\begin{aligned}
& P_{i}-E_{i}^{T} U_{i} E_{i}>0, \quad M_{1 i}>0
\end{aligned}
$$

where

$$
H_{i}^{\prime}=P_{i}\left(A_{i}+A_{d i}\right)+\left(A_{d i}+A_{i}\right)^{T} P_{i}+S_{1}+R_{1}+h Q_{i}+E_{i}^{T} U_{i} E_{i}
$$

for any fixed $i \in I_{0}$ and for all $A_{j} \in \mathcal{A}, b_{j} \in \mathcal{B}, a_{j} \in \mathcal{E}, A_{d j} \in \mathcal{A}_{d}$, such that

$$
\begin{gathered}
\bar{S}_{1}=\left[\begin{array}{cc}
S_{1} & S_{3} \\
S_{3}^{T} & S_{2}
\end{array}\right], \quad \bar{S}_{3}=\left[\begin{array}{l}
S_{31} \\
S_{32}
\end{array}\right], S_{1} \in R^{n \times n}, S_{2} \in R, S_{3} \in R^{n \times 1}, S_{31} \in R^{n \times(n+1)}, S_{32} \in R^{1 \times(n+1)} \\
\bar{R}=\left[\begin{array}{ll}
R_{1} & R_{3} \\
R_{3}^{T} & R_{2}
\end{array}\right], R_{1} \in R^{n \times n}, R_{2} \in R, R_{3} \in R^{n \times 1}
\end{gathered}
$$

satisfying

$$
\left[\begin{array}{cc}
\bar{S}_{1} & \bar{S}_{3} \\
\bar{S}_{3}^{T} & \bar{S}_{2}
\end{array}\right]>0, \quad \bar{R}>0
$$

for $\bar{S}_{1}, \bar{S}_{2}$ and $\bar{S}_{3}$. Furthermore, let the following inequalities hold

$$
\left[\begin{array}{cccc}
\bar{H}_{i}^{\prime} & \bar{P}_{i} & h \bar{P}_{i} \bar{A}_{d i} & \bar{S}_{3} \\
* & -\bar{M}_{1 i} & 0 & 0 \\
* & * & -h \bar{M}_{2 i} & 0 \\
* & * & * & \bar{S}_{2}-(1-d) \bar{R}
\end{array}\right]<0
$$




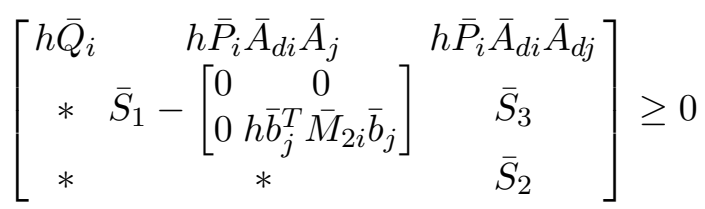

$$
\begin{aligned}
& \bar{P}_{i}-\bar{E}_{i}^{T} \bar{W}_{i} \bar{E}_{i}>0, \quad \bar{M}_{k i}>0, k=1,2
\end{aligned}
$$

for any fixed $i \in I_{1}$ and for all $\bar{A}_{j} \in \overline{\mathcal{A}}, \bar{b}_{j} \in \overline{\mathcal{B}}, \bar{A}_{d j} \in \overline{\mathcal{A}}_{d}$, where

$$
\bar{H}_{i}^{\prime}:=\bar{P}_{i}\left(\bar{A}_{i}+\bar{A}_{d i}\right)+\left(\bar{A}_{d i}+\bar{A}_{i}\right)^{T} \bar{P}_{i}+\bar{S}_{1}+\bar{R}+\left[\begin{array}{ll}
0 & 0 \\
0 & \bar{b}_{i}^{T} \bar{M}_{1 i} \bar{b}_{i}
\end{array}\right]+h \bar{Q}_{i}+\bar{E}_{i}^{T} \bar{U}_{i} \bar{E}_{i}
$$

Assume also that for all $i \in I$ and $k \in \mathcal{N}_{i}$,

$$
\begin{aligned}
F_{i k}^{T}\left(P_{i}-P_{k}\right) F_{i k} & =0 \\
F_{i k}^{T}\left(P_{i}-P_{k}\right) l_{i k}+F_{i k}^{T}\left(q_{i}-q_{k}\right) & =0 \\
l_{i k}^{T}\left(P_{i}-P_{k}\right) l_{i k}+2\left(q_{i}-q_{k}\right)^{T} l_{i k}+\left(r_{i}-r_{k}\right) & =0
\end{aligned}
$$

where $\bar{P}_{i}:=\left[\begin{array}{cc}P_{i} & q_{i} \\ q_{i}^{T} & r_{i}\end{array}\right]$, for all $i \in I$.

Under conditions (2), (3) and (9)-(18), every piecewise $C^{1}$ trajectory $x(t)$, governed by (5) for $t \geq 0$, tends to zero asymptotically in the absence of sliding modes.

Proof: Define the candidate Lyapunov-Krasovsky functional

$$
\bar{V}_{i}=\bar{V}_{1 i}+\bar{V}_{2 i}+\bar{V}_{3 i}
$$

where, for $x(t) \in X_{i}, i \in I_{1}$

$$
\begin{gathered}
\bar{V}_{1 i}=\bar{x}^{T}(t) \bar{P}_{i} \bar{x}(t) \\
\bar{V}_{2 i}=\int_{t-\tau(t)}^{t} \bar{x}^{T}(s) \bar{R} \bar{x}(s) d s \\
\bar{V}_{3 i}=h^{-1} \int_{-h}^{0} \int_{t+s}^{t}\left[\begin{array}{c}
\bar{x}(\theta) \\
\bar{x}(\theta-\tau(\theta))
\end{array}\right]^{T}\left[\begin{array}{cc}
\bar{S}_{1} & \bar{S}_{3} \\
\bar{S}_{3}^{T} & \bar{S}_{2}
\end{array}\right]\left[\begin{array}{c}
\bar{x}(\theta) \\
\bar{x}(\theta-\tau(\theta))
\end{array}\right] d \theta d s
\end{gathered}
$$

The conditions that guarantee the continuity of the Lyapunov function at the boundaries are give in (18ac), and can be obtained directly using the same approach as the one in (Rodrigues and How 2003). Note that the candidate Lyapunov functional is positive definite because of (16) and (13). Applying Leibnitz integral rule and using (2), the derivative of this Lyapunov functional will be obtained as 


$$
\begin{aligned}
\dot{\bar{V}}_{i} \leq & 2 \bar{x}(t)^{T} \bar{P}_{i} \dot{\bar{x}}(t)+\bar{x}^{T}(t) \bar{R} \bar{x}(t)-\bar{x}^{T}(t-\tau(t))(1-d) \bar{R} \bar{x}(t-\tau(t))+\bar{x}^{T}(t) \bar{S}_{1} \bar{x}(t) \\
& +\bar{x}^{T}(t-\tau(t)) \bar{S}_{2} \bar{x}(t-\tau(t))+2 \bar{x}^{T}(t) \bar{S}_{3} \bar{x}(t-\tau(t)) \\
& -h^{-1} \int_{t-\tau(t)}^{t}\left[\begin{array}{c}
\bar{x}(s) \\
\bar{x}(s-\tau(s))
\end{array}\right]^{T}\left[\begin{array}{cc}
\bar{S}_{1} & \bar{S}_{3} \\
\bar{S}_{3}^{T} & \bar{S}_{2}
\end{array}\right]\left[\begin{array}{c}
\bar{x}(s) \\
\bar{x}(s-\tau(s))
\end{array}\right] d s
\end{aligned}
$$

Substituting (7) in (21) leads to

$$
\begin{aligned}
\dot{\bar{V}}_{i} \leq & 2 \bar{x}^{T}(t) \bar{P}_{i}\left(\bar{A}_{i}+\bar{A}_{d i}\right) \bar{x}(t)+2 \bar{x}^{T}(t) \bar{P}_{i} \bar{b}_{i} 1(t-\tau(t)) \\
& +\bar{x}^{T}(t)\left(\bar{S}_{1}+\bar{R}\right) \bar{x}(t)+2 \bar{x}^{T}(t) \bar{S}_{3} \bar{x}(t-\tau(t))+\bar{x}^{T}(t-\tau(t))\left(\bar{S}_{2}-(1-d) \bar{R}\right) \bar{x}(t-\tau(t)) \\
& -2 \bar{x}^{T}(t) \bar{P}_{i} \bar{A}_{d i} \int_{t-\tau(t)}^{t} \bar{A}_{j(s)} \bar{x}(s) d s-2 \bar{x}^{T}(t) \bar{P}_{i} \bar{A}_{d i} \int_{t-\tau(t)}^{t} \bar{A}_{d j(s)} \bar{x}(s-\tau(s)) d s \\
& -2 \bar{x}^{T}(t) \bar{P}_{i} \bar{A}_{d i} \int_{t-\tau(t)}^{t} \bar{b}_{j(s)} 1(s-\tau(s)) d s \\
& -h^{-1} \int_{t-\tau(t)}^{t}\left[\begin{array}{c}
\bar{x}(s) \\
\bar{x}(s-\tau(s))
\end{array}\right]^{T}\left[\begin{array}{cc}
\bar{S}_{1} & \bar{S}_{3} \\
\bar{S}_{3}^{T} & \bar{S}_{2}
\end{array}\right]\left[\begin{array}{c}
\bar{x}(s) \\
\bar{x}(s-\tau(s))
\end{array}\right] d s
\end{aligned}
$$

Now, considering positive-definite matrices $\bar{M}_{k i}, k=1,2, i \in I_{1}$ using Lemma 1 and the inequalities (2), (22) yields

$$
\begin{aligned}
\dot{\bar{V}}_{i} \leq & 2 \bar{x}^{T}(t) \bar{P}_{i}\left(\bar{A}_{i}+\bar{A}_{d i}\right) \bar{x}(t)+\bar{x}^{T}(t) \bar{P}_{i} \bar{M}_{1 i}^{-1} \bar{P}_{i} \bar{x}(t)+\bar{b}_{i}^{T} \bar{M}_{1 i} \bar{b}_{i} 1(t-\tau(t)) \\
& +\bar{x}^{T}(t)\left(\bar{S}_{1}+\bar{R}\right) \bar{x}(t)+\bar{x}^{T}(t-\tau(t))\left(\bar{S}_{2}-(1-d) \bar{R}\right) \bar{x}(t-\tau(t))+2 \bar{x}^{T}(t) \bar{S}_{3} \bar{x}(t-\tau(t)) \\
& -2 \bar{x}^{T}(t) \bar{P}_{i} \bar{A}_{d i} \int_{t-\tau(t)}^{t} \bar{A}_{j(s)} \bar{x}(s) d s-2 \bar{x}^{T}(t) \bar{P}_{i} \bar{A}_{d i} \int_{t-\tau(t)}^{t} \bar{A}_{d j(s)} \bar{x}(s-\tau(s)) d s \\
& +h \bar{x}^{T}(t) \bar{P}_{i} \bar{A}_{d i} \bar{M}_{2 i}^{-1} \bar{A}_{d i}^{T} \bar{P}_{i} \bar{x}(t)+\int_{t-\tau(t)}^{t} \bar{b}_{j(s)}^{T} \bar{M}_{2 i} \bar{b}_{j(s)} 1(s-\tau(s)) d s \\
& -h^{-1} \int_{t-\tau(t)}^{t}\left[\begin{array}{c}
\bar{x}(s) \\
\bar{x}(s-\tau(s))
\end{array}\right]^{T}\left[\begin{array}{c}
\bar{S}_{1} \bar{S}_{3} \\
\bar{S}_{3}^{T} \\
\bar{S}_{2}
\end{array}\right]\left[\begin{array}{c}
\bar{x}(s) \\
\bar{x}(s-\tau(s))
\end{array}\right] d s
\end{aligned}
$$

Note that from (2), there always exists a symmetric positive semi-definite matrix $\bar{Q}_{i}$ such that

$$
h \bar{x}^{T}(t) \bar{Q}_{i} \bar{x}(t)-\int_{t-\tau(t)}^{t} \bar{x}^{T}(t) \bar{Q}_{i} \bar{x}(t) d s \geq 0
$$

Define now

$$
\begin{aligned}
& \bar{H}_{i}:=\bar{P}_{i}\left(\bar{A}_{i}+\bar{A}_{d i}\right)+\left(\bar{A}_{d i}+\bar{A}_{i}\right)^{T} \bar{P}_{i}+\bar{S}_{1}+\bar{R}+\left[\begin{array}{ll}
0 & 0 \\
0 & \bar{b}_{i}^{T} \bar{M}_{1 i} \bar{b}_{i}
\end{array}\right]+h \bar{Q}_{i} \\
& \bar{Z}_{i}:=\left[\begin{array}{cc}
\bar{H}_{i}+\bar{P}_{i} \bar{M}_{1 i}^{-1} \bar{P}_{i}+h \bar{P}_{i} \bar{A}_{d i} \bar{M}_{2 i}^{-1} \bar{A}_{d i}^{T} \bar{P}_{i} & \bar{S}_{3} \\
\bar{S}_{3}^{T} & \bar{S}_{2}-(1-d) \bar{R}
\end{array}\right]
\end{aligned}
$$




$$
\bar{Y}_{j(s)}:=-\left[\begin{array}{ccc}
h \bar{Q}_{i} & h \bar{P}_{i} \bar{A}_{d i} \bar{A}_{j(s)} & h \bar{P}_{i} \bar{A}_{d i} \bar{A}_{d j(s)} \\
* & \bar{S}_{1}-\left[\begin{array}{cc}
0 & 0 \\
0 h \bar{b}_{j(s)}^{T} & \bar{M}_{2 i} \bar{b}_{j(s)}
\end{array}\right] & \bar{S}_{3} \\
* & \multirow{2}{*}{*} & \bar{S}_{2}
\end{array}\right]
$$

Then by adding inequality (24) to the right hand side of (23) and considering (25a-c), one can write the following for $x(t) \in X_{i}, i \in I_{1}$

$$
\begin{aligned}
\dot{\bar{V}}_{i} \leq & \bar{\xi}^{T}(t, \tau(t)) \bar{Z}_{i} \bar{\xi}(t, \tau(t)) \\
& +h^{-1} \int_{t-\tau(t)}^{t} \bar{\eta}^{T}(t, s, \tau(s)) \bar{Y}_{j(s)} \bar{\eta}(t, s, \tau(s)) d s
\end{aligned}
$$

where $\bar{\xi}(t, \tau(t))=\left[\bar{x}^{T}(t), \bar{x}^{T}(t-\tau(t))\right]^{T}$ and $\bar{\eta}(t, s, \tau(s))=\left[\bar{x}^{T}(t), \bar{x}^{T}(s), \bar{x}^{T}(s-\tau(s))\right]^{T}$. Note that $(14)$ and (16) imply

$$
\bar{\xi}^{T}(\cdot)\left(\bar{Z}_{i}+\tilde{\bar{E}}_{i}^{T} \tilde{\bar{U}}_{i} \tilde{\bar{E}}_{i}\right) \bar{\xi}(\cdot)<0
$$

using the Schur complement, where $\tilde{\bar{U}}_{i}=\operatorname{diag}\left[\bar{U}_{i}, 0\right], \tilde{\bar{E}}_{i}=\left[\bar{E}_{i}, 0\right]$, and $\bar{U}_{i}$ has only non-negative entries. Note also that from (3), the inequality $\bar{E}_{i} \bar{x}(t) \geq 0$ holds for $x(t) \in X_{i}$. This leads to

$$
\tilde{\bar{E}}_{i} \bar{\xi} \geq 0, \forall x(t) \in X_{i}, i \in I_{1}
$$

and consequently it follows that

$$
\bar{\xi}^{T}(\cdot) \tilde{\bar{E}}_{i}^{T} \tilde{\bar{U}}_{i} \tilde{\bar{E}}_{i} \bar{\xi}(\cdot) \geq 0, \quad x(t) \in X_{i}, \quad i \in I_{1}
$$

Therefore, the relations (3), (16) and (14) imply $\bar{\xi}^{T}(\cdot) \bar{Z}_{i} \bar{\xi}(\cdot)<0$, for all $x(t) \in X_{i}, i \in I_{1}$. Furthermore, (15) implies $\bar{Y}_{j(s)} \leq 0$ and from $(26), \dot{\bar{V}}_{i}<0, x(t) \in X_{i}, i \in I_{1}$. A similar procedure can be repeated for the case when the switching index belongs to $I_{0}$ leading to (9)-(11) and $\dot{V}_{i}<0, x(t) \in X_{i}, i \in I_{0}$. Thus the system is asymptotically stable.

Remark 1: Theorem 1 assumes the absence of sliding modes. To avoid sliding modes at the boundaries the following conditions can be added. Let the set $\left\{x \in R^{n} \mid \sigma_{i k}=c_{i k}^{T} x-d_{i k}=0\right\}$ denote the sliding surface between the cells $X_{i}$ and $X_{k}$. According to (Rodrigues and How 2003), $\dot{\sigma}_{i k}$ must be continuous across the boundary described in (4), which yields

$$
\begin{aligned}
c_{i k}^{T} & {\left[A_{i}\left(F_{i k} s+l_{i k}\right)+A_{d i} x(t-\tau(t))+a_{i}+b_{i} 1(t-\tau(t))\right] } \\
& =c_{i k}^{T}\left[A_{k}\left(F_{i k} s+l_{i k}\right)+A_{d k} x(t-\tau(t))+a_{k}+b_{k} 1(t-\tau(t))\right]
\end{aligned}
$$

for all $s \in R^{n-1}, k \in \mathcal{N}_{i}$. The above equation can be rewritten as follows

$$
\begin{aligned}
c_{i k}^{T}\left(A_{i}-A_{k}\right) F_{i k} & =0 \\
c_{i k}^{T}\left(A_{d i}-A_{d k}\right) & =0 \\
c_{i k}^{T}\left[\left(A_{i}-A_{k}\right) l_{i k}+\left(a_{i}-a_{k}\right)\right] & =0 \\
c_{i k}^{T}\left(b_{i}-b_{k}\right) & =0
\end{aligned}
$$

Remark 2: Using a procedure similar to the one presented here, one can apply the results of (Mondie and Kharitonov 2005) and define the following Lyapunov-Krasovsky functional 


$$
\begin{aligned}
\bar{V}_{i}^{\prime}= & \bar{x}^{T}(t) \bar{P}_{i} \bar{x}(t)+\int_{t-\tau(t)}^{t} \bar{x}^{T}(s) e^{\beta(s-t)} \bar{R} \bar{x}(s) d s \\
& +h^{-1} \int_{-h}^{0} \int_{t+s}^{t}\left[\begin{array}{c}
\bar{x}(\theta) \\
\bar{x}(\theta-\tau(\theta))
\end{array}\right]^{T} e^{\beta(\theta-t)}\left[\begin{array}{cc}
\bar{S}_{1} & \bar{S}_{3} \\
\bar{S}_{3}^{T} & \bar{S}_{2}
\end{array}\right]\left[\begin{array}{c}
\bar{x}(\theta) \\
\bar{x}(\theta-\tau(\theta))
\end{array}\right] d \theta d s
\end{aligned}
$$

to obtain the LMIs that determine the exponential stability of the system (5). It is to be noted that exponential stability typically is stronger than asymptotic stability, at the cost of more conservative LMIs.

\section{Robustness Analysis}

Consider now the system (1) and define the matrices $\tilde{A}_{i}=A_{i}+\Delta A_{i}, \tilde{A}_{d i}=A_{d i}+\Delta A_{d i}, \tilde{a}_{i}=a_{i}+\Delta a_{i}$, $\tilde{b}_{i}=b_{i}+\Delta b_{i}(i \in I)$ and

$$
\begin{gathered}
\overline{\tilde{A}}_{i}=\bar{A}_{i}+\Delta \bar{A}_{i}, \quad \Delta \bar{A}_{i}=\left[\begin{array}{cc}
\Delta A_{i} & \Delta a_{i} \\
0 & 0
\end{array}\right] \\
\overline{\tilde{A}}_{d i}=\bar{A}_{d i}+\Delta \bar{A}_{d i}, \quad \Delta \bar{A}_{d i}=\left[\begin{array}{cc}
\Delta A_{d i} 0 \\
0 & 0
\end{array}\right] \\
\overline{\tilde{b}}_{i}=\bar{b}_{i}+\Delta \bar{b}_{i}, \quad \Delta \bar{b}_{i}=\left[\begin{array}{c}
\Delta b_{i} \\
0
\end{array}\right] \\
\left.\Delta \overline{\mathcal{A}}=\left\{\begin{array}{c}
\Delta A_{j} \Delta a_{j} \\
0
\end{array}\right], \forall j \in I\right\}, \quad \Delta \mathcal{A}=\left\{\Delta A_{j}, \quad \forall j \in I\right\}, \quad \Delta \mathcal{E}=\left\{\Delta a_{j}, \quad \forall j \in I_{1}\right\} \\
\Delta \overline{\mathcal{B}}=\left\{\left[\begin{array}{c}
\Delta b_{j} \\
0
\end{array}\right], \forall j \in I_{1}\right\}, \quad \Delta \mathcal{B}=\left\{\Delta b_{j}, \forall j \in I_{1}\right\} \\
\Delta \overline{\mathcal{A}}_{d}=\left\{\left[\begin{array}{c}
\Delta A_{d j} \\
0 \\
0
\end{array}\right], \forall j \in I\right\}, \quad \Delta \mathcal{A}_{d}=\left\{\Delta A_{d j}, \forall j \in I\right\}
\end{gathered}
$$

Let $\|\cdot\|$ denote the 2-norm. The following bounds are assumed to be given for the norm of relevant matrices

$$
\begin{gathered}
\left\|\Delta A_{i}\right\| \leq \alpha_{i}, \quad\left\|\Delta A_{d i}\right\| \leq \beta_{i} \\
\left\|\Delta \bar{A}_{i}\right\| \leq \bar{\alpha}_{i}, \quad\left\|\Delta \bar{A}_{d i}\right\| \leq \bar{\beta}_{i}, \quad\left\|\Delta \bar{b}_{i}\right\| \leq \bar{\delta}_{i} \\
\max _{X \in \Delta \overline{\mathcal{A}}}\|X\| \leq \bar{\alpha}^{*}, \quad \max _{X \in \Delta \overline{\mathcal{A}}_{d}}\|X\|=\max _{X \in \Delta \mathcal{A}_{d}}\|X\| \leq \beta^{*}
\end{gathered}
$$




$$
\begin{gathered}
\max _{X \in \Delta \mathcal{A}}\|X\| \leq \alpha^{*}, \quad \max _{X \in \Delta \mathcal{E}}\|X\| \leq \gamma^{*} \\
\max _{X \in \Delta \overline{\mathcal{B}}}\|X\|=\max _{X \in \Delta \mathcal{B}}\|X\| \leq \delta^{*}
\end{gathered}
$$

The following theorem presents sufficient conditions for the stability of uncertain PWA systems described by (1).

Theorem 2: Consider symmetric matrices $\bar{U}_{i}, U_{i}$ and $\bar{W}_{i}, W_{i}$, where $\bar{U}_{i}, U_{i}$ and $\bar{W}_{i}, W_{i}$ are composed of non-negative entries. Then, the uncertain PWA time-delay system (1) is asymptotically stable in the absence of sliding modes, if (11), (16), (13) and (18a-c) hold, and there exist positive definite matrices $L_{k i}$, $k=1, \ldots, 10, \bar{L}_{k i},(k=1, \ldots, 9), M_{1 i}, i \in I_{0}$ and $\bar{M}_{p i}, p=1,2, i \in I_{1}$ such that

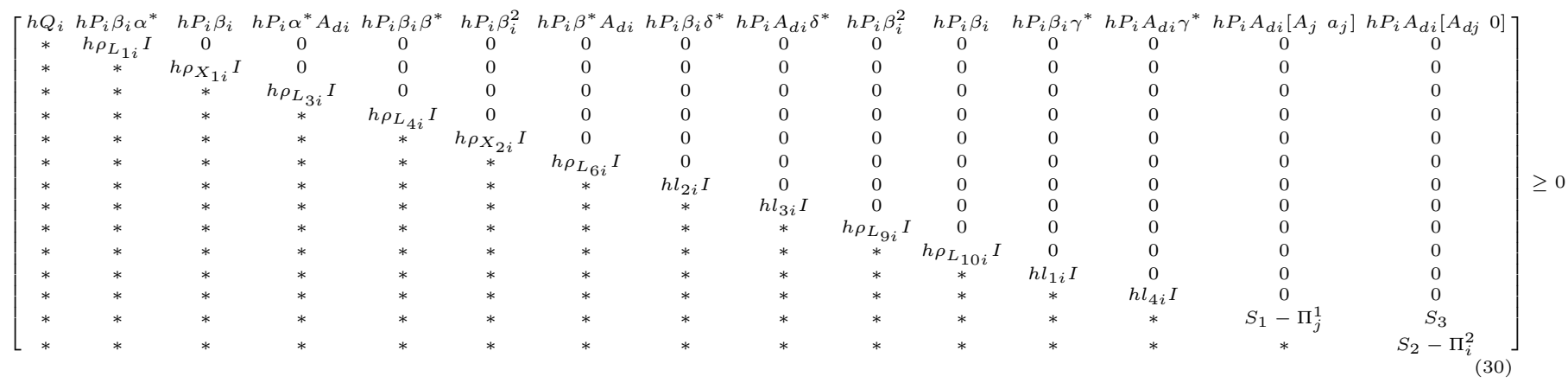

where

$$
\Pi_{j}^{1}=h\left(L_{1 i}+L_{2 i}+L_{3 i}+\left[\begin{array}{lc}
0 & 0 \\
0 & b_{j}^{T}\left(M_{1 i}+L_{9 i}\right) b_{j}
\end{array}\right]+\left[\begin{array}{lc}
0 & 0 \\
0 & a_{j}^{T} L_{10 i} a_{j}
\end{array}\right]\right), \quad \Pi_{i}^{2}=h\left(L_{4 i}+L_{5 i}+L_{6 i}\right)
$$

$\left[\begin{array}{ccccc}H_{i}^{\prime} & h P_{i} A_{d i} & P_{i} \alpha_{i} & P_{i} \beta_{i} & S_{31}+\left[0_{n \times n} R_{3}+S_{3}\right] \\ * & -h M_{1 i} & 0 & 0 & 0 \\ * & * & -\rho_{L_{7 i}} I & 0 & 0 \\ * & * & * & -\rho_{L_{8 i}} I & 0 \\ * & * & * & * & \bar{S}_{2}-(1-d) \bar{R}+\left[\begin{array}{cc}0_{n \times n} & 0_{n \times 1} \\ 0_{1 \times n} & S_{2}+R_{2}\end{array}\right]+\left[\begin{array}{c}0_{n \times(n+1)} \\ S_{32}\end{array}\right]+\left[0_{(n+1) \times n} S_{32}^{T}\right]\end{array}\right]<0$

$$
\left[\begin{array}{cc}
X_{1 i} & X_{1 i} A_{j} \\
* & L_{2 i}
\end{array}\right]>0, \quad\left[\begin{array}{cc}
X_{2 i} & X_{2 i} A_{d j} \\
* & L_{5 i}
\end{array}\right]>0
$$

for any fixed $i \in I_{0}$ and for all $A_{j} \in \mathcal{A}, b_{j} \in \mathcal{B}, a_{j} \in \mathcal{E}, A_{d j} \in \mathcal{A}_{d}$. In addition, let the following LMIs hold 


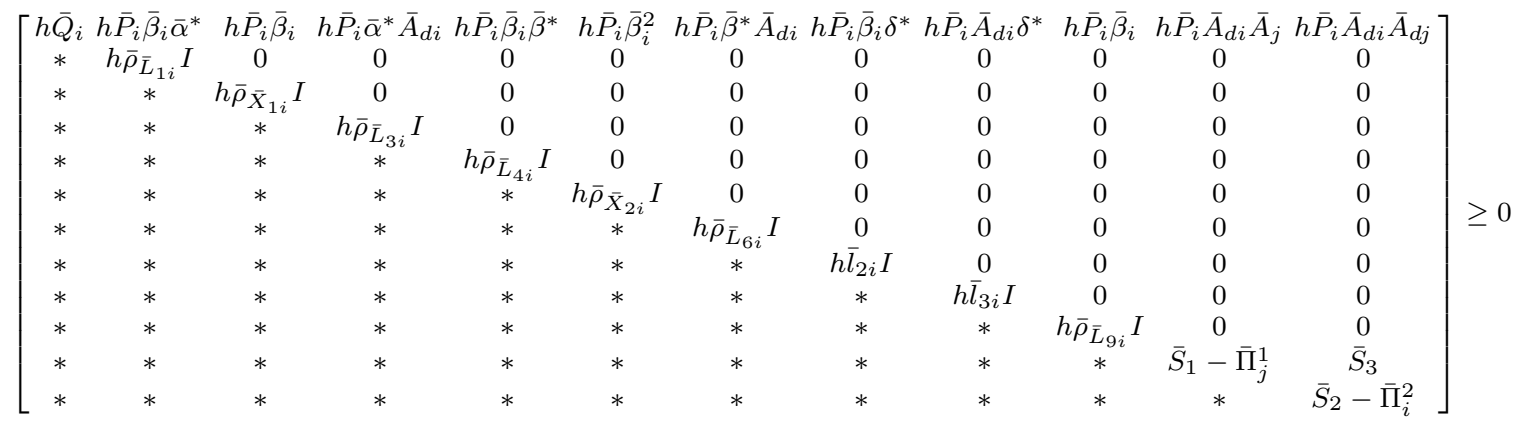

$$
\begin{aligned}
& \bar{\Pi}_{j}^{1}=h\left(\bar{L}_{1 i}+\bar{L}_{2 i}+\bar{L}_{3 i}+\left[\begin{array}{ll}
0 & 0 \\
0 & \bar{b}_{j}^{T}\left(\bar{M}_{2 i}+\bar{L}_{9 i}\right) \bar{b}_{j}
\end{array}\right]\right), \quad \bar{\Pi}_{i}^{2}=h\left(\bar{L}_{4 i}+\bar{L}_{5 i}+\bar{L}_{6 i}\right) \\
& {\left[\begin{array}{ccccccc}
\hat{\bar{H}}_{i} & \bar{P}_{i} & h \bar{P}_{i} \bar{A}_{d i} & \bar{P}_{i} \bar{\alpha}_{i} & \bar{P}_{i} \bar{\beta}_{i} & \bar{P}_{i} \bar{\delta}_{i} & \bar{S}_{3} \\
* & -\bar{M}_{1 i} & 0 & 0 & 0 & 0 & 0 \\
* & * & -h \bar{M}_{2 i} & 0 & 0 & 0 & 0 \\
* & * & * & -\bar{\rho}_{\bar{L}_{7 i}} I & 0 & 0 & 0 \\
* & * & * & * & -\bar{\rho}_{\bar{L}_{8 i}} I & 0 & 0 \\
* & * & * & * & * & -\bar{l}_{1 i} I & 0 \\
* & * & * & * & * & * & \bar{S}_{2}-(1-d) \bar{R}
\end{array}\right]<0} \\
& {\left[\begin{array}{cc}
\bar{X}_{1 i} & \bar{X}_{1 i} \bar{A}_{j} \\
* & \bar{L}_{2 i}
\end{array}\right]>0, \quad\left[\begin{array}{cc}
\bar{X}_{2 i} & \bar{X}_{2 i} \bar{A}_{d j} \\
* & \bar{L}_{5 i}
\end{array}\right]>0}
\end{aligned}
$$

for any fixed $i \in I_{1}$ and for all $\bar{A}_{j} \in \overline{\mathcal{A}}, \bar{b}_{j} \in \overline{\mathcal{B}}, \bar{A}_{d j} \in \overline{\mathcal{A}}_{d}$, where the following inequalities are satisfied

$$
\begin{gathered}
\rho_{L_{k i}}>0, \quad L_{k i}-\rho_{L_{k i}} I>0, \quad k=1,3,4,6,7, \ldots, 10 \quad \forall i \in I_{0} \\
\bar{\rho}_{\bar{L}_{k i}}>0, \quad \bar{L}_{k i}-\bar{\rho}_{\bar{L}_{k i}} I>0, \quad k=1,3,4,6,7,8,9 \quad \forall i \in I_{1} \\
\rho_{X_{k i}}>0, \quad \rho_{X_{k i}} I-X_{k i}<0, \quad k=1 \text { and } 2 \quad \forall i \in I_{0} \\
\bar{\rho}_{\bar{X}_{k i}}>0, \quad \bar{\rho}_{\bar{X}_{k i}} I-\bar{X}_{k i}<0, \quad k=1 \text { and } 2 \quad \forall i \in I_{1}
\end{gathered}
$$

where

$$
\hat{H}_{i}=H_{i}^{\prime}+L_{7 i}+L_{8 i}+\left[\begin{array}{ll}
0 & 0 \\
0 & h l_{1 i}+h l_{4 i}
\end{array}\right], \quad \hat{\bar{H}}_{i}=\bar{H}_{i}^{\prime}+\bar{L}_{7 i}+\bar{L}_{8 i}+\left[\begin{array}{l}
0 \\
0 \\
0 \bar{l}_{1 i}+h \bar{l}_{2 i}+h \bar{l}_{3 i}
\end{array}\right]
$$

(note that $H_{i}^{\prime}$ and $\bar{H}_{i}^{\prime}$ are defined in (12) and (17), respectively).

Proof: The proof follows the steps of the proof of Theorem 1 , after replacing $\bar{A}_{i}$ with, $\overline{\tilde{A}}_{i}, \bar{A}_{d i}$ with $\overline{\tilde{A}}_{d i}$ and $\bar{b}_{i}$ with $\overline{\tilde{b}}_{i}$. 
Doing this, expression (26) becomes for $x(t) \in X_{i}, i \in I_{1}$

$$
\begin{aligned}
\dot{\bar{V}} \leq \bar{\xi}^{T}(t, \tau(t)) \bar{Z}_{i} \bar{\xi}(t, \tau(t)) \\
\quad+h^{-1} \int_{t-\tau(t)}^{t} \bar{\eta}^{T}(t, s, \tau(s)) \bar{Y}_{j(s)} \bar{\eta}(t, s, \tau(s)) d s \\
\quad+2 \bar{x}^{T} \bar{P}_{i}\left[\left(\Delta \bar{A}_{i}+\Delta \bar{A}_{d i}\right) \bar{x}(t)+\Delta \bar{b}_{i} 1(t-\tau(t))-\Delta \bar{A}_{d i} \int_{t-\tau(t)}^{t} \Delta \bar{A}_{j(s)} \bar{x}(s) d s\right. \\
\quad-\Delta \bar{A}_{d i} \int_{t-\tau(t)}^{t} \bar{A}_{j(s)} \bar{x}(s) d s-\bar{A}_{d i} \int_{t-\tau(t)}^{t} \Delta \bar{A}_{j(s)} \bar{x}(s) d s \\
\quad-\Delta \bar{A}_{d i} \int_{t-\tau(t)}^{t} \Delta \bar{A}_{d j(s)} \bar{x}(s-\tau(s)) d s-\bar{A}_{d i} \int_{t-\tau(t)}^{t} \Delta \bar{A}_{d j(s)} \bar{x}(s-\tau(s)) d s \\
\quad-\Delta \bar{A}_{d i} \int_{t-\tau(t)}^{t} \bar{b}_{j(s)} 1(s-\tau(s)) d s-\Delta \bar{A}_{d i} \int_{t-\tau(t)}^{t} \Delta \bar{b}_{j(s)} 1(s-\tau(s)) d s \\
\left.\quad-\bar{A}_{d i} \int_{t-\tau(t)}^{t} \Delta \bar{b}_{j(s)} 1(s-\tau(s)) d s-\Delta \bar{A}_{d i} \int_{t-\tau(t)}^{t} \bar{A}_{d j(s)} \bar{x}(s-\tau(s)) d s\right]
\end{aligned}
$$

The objective now is to find upper bounds to all terms of (40). Defining positive definite matrices $\bar{L}_{7 i}, \bar{L}_{8 i}$, a positive constant $\bar{l}_{1 i}$ and using Lemma 1 , yields

$$
\begin{aligned}
& 2 \bar{x}^{T}(t) \bar{P}_{i}\left[\left(\Delta \bar{A}_{i}+\Delta \bar{A}_{d i}\right) \bar{x}(t)+\Delta \bar{b}_{i} 1(t-\tau(t))\right] \leq \bar{x}^{T}(t) \bar{P}_{i} \Delta \bar{A}_{i} \bar{L}_{7 i}^{-1} \Delta \bar{A}_{i}^{T} \bar{P}_{i} \bar{x}(t) \\
& \quad+\bar{x}^{T}(t) \bar{P}_{i} \Delta \bar{A}_{d i} \bar{L}_{8 i}^{-1} \Delta \bar{A}_{d i}^{T} \bar{P}_{i} \bar{x}(t)+\bar{x}^{T}(t) \bar{P}_{i} \Delta \bar{b}_{i} \bar{l}_{1 i}^{-1} \Delta \bar{b}_{i}^{T} \bar{P}_{i} \bar{x}(t)+\bar{x}^{T}(t) \bar{L}_{7 i} \bar{x}(t)+\bar{x}^{T}(t) \bar{L}_{8 i} \bar{x}(t)+\bar{l}_{1 i} 1(t-\tau(t))
\end{aligned}
$$

Considering the fact that, $\lambda_{\text {min }}^{-1}\left(\bar{L}_{k i}\right)=\lambda_{\max }\left(\bar{L}_{k i}^{-1}\right), k=1, \ldots, 9$, and $\left\|\bar{x}^{T}(t) \bar{P}_{i}\right\|^{2}=\bar{x}^{T}(t) \bar{P}_{i} \bar{P}_{i} \bar{x}(t)$, expression (41) can be rewritten as

$$
\begin{aligned}
& 2 \bar{x}^{T}(t) \bar{P}_{i}\left[\left(\Delta \bar{A}_{i}+\Delta \bar{A}_{d i}\right) \bar{x}(t)+\Delta \bar{b}_{i} 1(t-\tau(t))\right] \leq \bar{x}^{T}(t) \bar{P}_{i}\left(\bar{\alpha}_{i}^{2} \lambda_{\min }^{-1}\left(\bar{L}_{7 i}\right)+\bar{\beta}_{i}^{2} \lambda_{\min }^{-1}\left(\bar{L}_{8 i}\right)+\bar{\delta}_{i}^{2} \bar{l}_{1 i}^{-1}\right) \bar{P}_{i} \bar{x}(t) \\
& \quad \bar{x}^{T}(t)\left(\bar{L}_{7 i}+\bar{L}_{8 i}\right) \bar{x}(t)+\bar{l}_{1 i}
\end{aligned}
$$

Defining positive definite matrices $\bar{L}_{k i}, k=1,3,4,6,9$, positive constants $\bar{l}_{m i}, m=2,3$ and following the same procedure as stated above, it can be shown that

$$
\begin{gathered}
-2 \bar{x}^{T}(t) \bar{P}_{i} \Delta \bar{A}_{d i} \int_{t-\tau(t)}^{t} \Delta \bar{A}_{j(s)} \bar{x}(s) d s \leq \int_{t-\tau(t)}^{t} \bar{x}^{T}(s) \bar{L}_{1 i} \bar{x}(s) d s \\
+\int_{t-\tau(t)}^{t} \bar{x}^{T}(t) \bar{P}_{i} \bar{\beta}_{i}^{2}\left(\bar{\alpha}^{*}\right)^{2} \lambda_{\min }^{-1}\left(\bar{L}_{1 i}\right) \bar{P}_{i} \bar{x}(t) d s \\
-2 \bar{x}^{T}(t) \bar{P}_{i} \bar{A}_{d i} \int_{t-\tau(t)}^{t} \Delta \bar{A}_{j(s)} \bar{x}(s) d s \leq \int_{t-\tau(t)}^{t} \bar{x}^{T}(s) \bar{L}_{3 i} \bar{x}(s) d s \\
+\int_{t-\tau(t)}^{t} \bar{x}^{T}(t) \bar{P}_{i} \bar{A}_{d i}\left(\bar{\alpha}^{*}\right)^{2} \lambda_{\min }^{-1}\left(\bar{L}_{3 i}\right) \bar{A}_{d i}^{T} \bar{P}_{i} \bar{x}(t) d s
\end{gathered}
$$




$$
\begin{aligned}
& -2 \bar{x}^{T}(t) \bar{P}_{i} \Delta \bar{A}_{d i} \int_{t-\tau(t)}^{t} \Delta \bar{A}_{d j(s)} \bar{x}(s-\tau(s)) d s \leq \int_{t-\tau(t)}^{t} \bar{x}^{T}(s-\tau(s)) \bar{L}_{4 i} \bar{x}(s-\tau(s)) d s \\
& +\int_{t-\tau(t)}^{t} \bar{x}^{T}(t) \bar{P}_{i} \bar{\beta}_{i}^{2}\left(\bar{\beta}^{*}\right)^{2} \lambda_{\min }^{-1}\left(\bar{L}_{4 i}\right) \bar{P}_{i} \bar{x}(t) d s \\
& -2 \bar{x}^{T}(t) \bar{P}_{i} \bar{A}_{d i} \int_{t-\tau(t)}^{t} \Delta \bar{A}_{d j(s)} \bar{x}(s-\tau(s)) d s \leq \int_{t-\tau(t)}^{t} \bar{x}^{T}(s-\tau(s)) \bar{L}_{6 i} \bar{x}(s-\tau(s)) d s \\
& +\int_{t-\tau(t)}^{t} \bar{x}^{T}(t) \bar{P}_{i} \bar{A}_{d i}\left(\bar{\beta}^{*}\right)^{2} \lambda_{\min }^{-1}\left(\bar{L}_{6 i}\right) \bar{A}_{d i}^{T} \bar{P}_{i} \bar{x}(t) d s \\
& -2 \bar{x}^{T}(t) \bar{P}_{i} \Delta \bar{A}_{d i} \int_{t-\tau(t)}^{t} \bar{b}_{j(s)} 1(s-\tau(s)) d s \leq \int_{t-\tau(t)}^{t} \bar{b}_{j(s)}^{T} \bar{L}_{9 i} \bar{b}_{j(s)} \overline{1}(s-\tau(s)) d s \\
& +\int_{t-\tau(t)}^{t} \bar{x}^{T}(t) \bar{P}_{i} \bar{\beta}_{i}^{2} \lambda_{\min }^{-1}\left(\bar{L}_{9 i}\right) \bar{P}_{i} \bar{x}(t) d s \\
& -2 \bar{x}^{T}(t) \bar{P}_{i} \Delta \bar{A}_{d i} \int_{t-\tau(t)}^{t} \Delta \bar{b}_{j(s)} 1(s-\tau(s)) d s \leq \int_{t-\tau(t)}^{t} \bar{l}_{2 i} 1(s-\tau(s)) d s \\
& +\int_{t-\tau(t)}^{t} \bar{x}^{T}(t) \bar{P}_{i}\left(\delta^{*}\right)^{2} \bar{\beta}_{i}^{2} \bar{l}_{2 i}^{-1} \bar{P}_{i} \bar{x}(t) d s \\
& -2 \bar{x}^{T}(t) \bar{P}_{i} \bar{A}_{d i} \int_{t-\tau(t)}^{t} \Delta \bar{b}_{j(s)} 1(s-\tau(s)) d s \leq \int_{t-\tau(t)}^{t} \bar{l}_{3 i} 1(s-\tau(s)) d s \\
& +\int_{t-\tau(t)}^{t} \bar{x}^{T}(t) \bar{P}_{i} \bar{A}_{d i}\left(\delta^{*}\right)^{2} \bar{l}_{3 i}^{-1} \bar{A}_{d i}^{T} \bar{P}_{i} \bar{x}(t) d s \\
& -2 \bar{x}^{T}(t) \bar{P}_{i} \Delta \bar{A}_{d i} \int_{t-\tau(t)}^{t} \bar{A}_{j(s)} \bar{x}(s) d s \leq \int_{t-\tau(t)}^{t} \bar{x}^{T}(s) \bar{L}_{2 i} \bar{x}(s) d s \\
& +\int_{t-\tau(t)}^{t} \bar{x}^{T}(t) \bar{P}_{i} \Delta \bar{A}_{d i}\left[\bar{A}_{j(s)} \bar{L}_{2 i}^{-1} \bar{A}_{j(s)}^{T}\right] \Delta \bar{A}_{d i}^{T} \bar{P}_{i} \bar{x}(t) d s
\end{aligned}
$$

If (35) is verified then there exist positive definite matrices $\bar{X}_{1 i}, \bar{L}_{2 i}$ and $\bar{X}_{2 i}, \bar{L}_{5 i}$ such that (Da et al. 2000)

$$
\bar{A}_{j(s)} \bar{L}_{2 i}^{-1} \bar{A}_{j(s)}^{T}<\bar{X}_{1 i}^{-1} \text { and } \bar{A}_{d j(s)} \bar{L}_{5 i}^{-1} \bar{A}_{d j(s)}^{T}<\bar{X}_{2 i}^{-1}
$$


Using (51), and the fact that $\lambda_{\max }\left(\bar{X}_{k i}^{-1}\right)=\lambda_{\min }^{-1}\left(\bar{X}_{k i}\right), k=1,2$ in inequality (50) yields

$$
\begin{gathered}
-2 \bar{x}^{T}(t) \bar{P}_{i} \Delta \bar{A}_{d i} \int_{t-\tau(t)}^{t} \bar{A}_{j(s)} \bar{x}(s) d s \leq \int_{t-\tau(t)}^{t} \bar{x}^{T}(s) \bar{L}_{2 i} \bar{x}(s) d s \\
+\int_{t-\tau(t)}^{t} \bar{x}^{T}(t) \bar{P}_{i} \bar{\beta}_{i}^{2} \lambda_{\min }^{-1}\left(\bar{X}_{1 i}\right) \bar{P}_{i} \bar{x}(t) d s
\end{gathered}
$$

Finally, applying the above argument to the last term of (40) and using (51) leads to

$$
\begin{gathered}
-2 \bar{x}^{T}(t) \bar{P}_{i} \Delta \bar{A}_{d i} \int_{t-\tau(t)}^{t} \bar{A}_{d j(s)} \bar{x}(s-\tau(s)) d s \leq \int_{t-\tau(t)}^{t} \bar{x}^{T}(s-\tau(s)) \bar{L}_{5 i} \bar{x}(s-\tau(s)) d s \\
+\int_{t-\tau(t)}^{t} \bar{x}^{T}(t) \bar{P}_{i} \bar{\beta}_{i}^{2} \lambda_{\min }^{-1}\left(\bar{X}_{2 i}\right) \bar{P}_{i} \bar{x}(t) d s
\end{gathered}
$$

Hence, substituting (42)-(49), (52) and (53) in (40) yields

$$
\begin{aligned}
& \dot{\bar{V}} \leq \bar{\xi}^{T}(t, \tau(t)) \bar{Z}_{i} \bar{\xi}(t, \tau(t)) \\
& +\bar{x}^{T}(t) \bar{P}_{i}\left(\lambda_{\text {min }}^{-1}\left(\bar{L}_{7 i}\right) \bar{\alpha}_{i}^{2}+\lambda_{\text {min }}^{-1}\left(\bar{L}_{8 i}\right) \bar{\beta}_{i}^{2}+\bar{\delta}_{1 i}^{-1}\right) \bar{P}_{i} \bar{x}(t) \\
& +\bar{x}^{T}(t)\left(\bar{L}_{7 i}+\bar{L}_{8 i}\right) \bar{x}(t)+\bar{l}_{1 i}+h \bar{l}_{2 i}+h \bar{l}_{3 i} \\
& +h^{-1} \int_{t-\tau(t)}^{t} \eta^{T}(t, s, \tau(s)) \bar{Y}_{j(s)} \eta(t, s, \tau(s)) d s \\
& +h^{-1} \int_{t-\tau(t)}^{t}\left(\overline { x } ^ { T } ( t ) h \overline { P } _ { i } \left[\lambda_{\min }^{-1}\left(\bar{L}_{1 i}\right) \bar{\beta}_{i}^{2}\left(\bar{\alpha}^{*}\right)^{2}+\lambda_{\text {min }}^{-1}\left(\bar{X}_{1 i}\right) \bar{\beta}_{i}^{2}\right.\right. \\
& +\lambda_{\min }^{-1}\left(\bar{L}_{3 i}\right)\left(\bar{\alpha}^{*}\right)^{2} \bar{A}_{d i} \bar{A}_{d i}^{T}+\lambda_{\text {min }}^{-1}\left(\bar{L}_{4 i}\right) \bar{\beta}_{i}^{2}\left(\bar{\beta}^{*}\right)^{2} \\
& +\lambda_{\text {min }}^{-1}\left(\bar{X}_{2 i}\right) \bar{\beta}_{i}^{2}+\lambda_{\text {min }}^{-1}\left(\bar{L}_{6 i}\right)\left(\bar{\beta}^{*}\right)^{2} \bar{A}_{d i} \bar{A}_{d i}^{T} \\
& \left.\left.+\left(\delta^{*}\right)^{2} \beta_{i}^{2} \bar{l}_{2 i}^{-1}+A_{d i} A_{d i}^{T}\left(\delta^{*}\right)^{2} \bar{l}_{3 i}^{-1}+\beta_{i}^{2} \lambda_{\min }^{-1}\left(\bar{L}_{9 i}\right)\right] \bar{P}_{i} \bar{x}(t)\right) d s \\
& +h^{-1} \int_{t-\tau(t)}^{t} h \bar{x}^{T}(s)\left(\bar{L}_{1 i}+\bar{L}_{2 i}+\bar{L}_{3 i}+\left[\begin{array}{ll}
0 & 0 \\
0 & b_{j(s)}^{T} \bar{L}_{9 i} b_{j(s)}
\end{array}\right]\right) \bar{x}(s) d s \\
& +h^{-1} \int_{t-\tau(t)}^{t} h \bar{x}^{T}(s-\tau(s))\left(\bar{L}_{4 i}+\bar{L}_{5 i}+\bar{L}_{6 i}\right) \bar{x}(s-\tau(s)) d s
\end{aligned}
$$

On the other hand, it is known that

$$
\lambda_{\min }\left(\bar{L}_{k i}\right) I \leq \bar{L}_{k i}, \quad \lambda_{\min }\left(\bar{X}_{p i}\right) I \leq \bar{X}_{p i}
$$

Inequalities (37) and (39) imply that one can find positive constants $\bar{\rho}_{L_{\bar{k} i}}$ and $\bar{\rho}_{\bar{X}_{p i}}$ such that (Mondie and Kharitonov 2005)

$$
\bar{\rho}_{\bar{L}_{k i}} I-\bar{L}_{k i}<0, \quad \bar{\rho}_{\bar{X}_{p i}} I-\bar{X}_{p i}<0
$$

where $k=1,3,4,6,7,8,9$ and $p=1,2$. This implies that $\bar{\rho}_{\bar{L}_{k i}}<\lambda_{\min }\left(\bar{L}_{k i}\right)$ and $\bar{\rho}_{\bar{X}_{p i}}<\lambda_{\min }\left(\bar{X}_{p i}\right)$. Let 
us denote

$$
\bar{\Omega}=\left[\begin{array}{ccc}
\bar{\Omega}_{11} & 0 & 0 \\
0 & \bar{\Omega}_{22} & 0 \\
0 & 0 & \bar{\Omega}_{33}
\end{array}\right], \quad \bar{\Gamma}=\left[\begin{array}{cc}
\bar{\Gamma}_{11} & 0 \\
0 & 0
\end{array}\right]
$$

where

$$
\begin{gathered}
\bar{\Omega}_{11}=\begin{array}{c}
h \bar{P}_{i}\left[\bar{\rho}_{\bar{L}_{1 i}}^{-1} \bar{\beta}_{i}^{2}\left(\bar{\alpha}^{*}\right)^{2}+\bar{\rho}_{\bar{X}_{1 i}}^{-1} \bar{\beta}_{i}^{2}+\bar{\rho}_{\bar{L}_{3 i}}^{-1}\left(\bar{\alpha}^{*}\right)^{2} \bar{A}_{d i} \bar{A}_{d i}^{T}\right. \\
+\bar{\rho}_{\bar{L}_{4 i}}^{-1} \bar{\beta}_{i}^{2}\left(\bar{\beta}^{*}\right)^{2}+\bar{\rho}_{\bar{X}_{2 i}}^{-1} \bar{\beta}_{i}^{2}+\bar{\rho}_{\bar{L}_{6 i}}^{-1}\left(\bar{\beta}^{*}\right)^{2} \bar{A}_{d i} \bar{A}_{d i}^{T} \\
\left.+\left(\delta^{*}\right)^{2} \beta_{i}^{2} \bar{l}_{2 i}^{-1}+\bar{A}_{d i} \bar{A}_{d i}^{T}\left(\delta^{*}\right)^{2} \bar{l}_{3 i}^{-1}+\bar{\beta}_{i}^{2} \bar{\rho}_{\bar{L}_{9 i}}^{-1}\right] \bar{P}_{i}
\end{array} \\
\bar{\Omega}_{22}=h\left(\bar{L}_{1 i}+\bar{L}_{2 i}+\bar{L}_{3 i}+\left[\begin{array}{ll}
0 & 0 \\
0 & b_{j(s)}^{T} \bar{L}_{9 i} b_{j(s)}
\end{array}\right]\right) \\
\bar{\Omega}_{33}=h\left(\bar{L}_{4 i}+\bar{L}_{5 i}+\bar{L}_{6 i}\right) \\
\bar{\Gamma}_{11}=\bar{P}_{i}\left(\bar{\rho}_{\bar{L}_{7 i}}^{-1} \bar{\alpha}_{i}^{2}+\bar{\rho}_{\bar{L}_{8 i}}^{-1} \bar{\beta}_{i}^{2}+\bar{\delta}_{i}^{2} \bar{l}_{1 i}^{-1}\right) \bar{P}_{i}+\bar{L}_{7 i}+\bar{L}_{8 i}+\left[\begin{array}{l}
0 \\
0 \bar{l}_{1 i}+h \bar{l}_{2 i}+h \bar{l}_{3 i}
\end{array}\right]
\end{gathered}
$$

Therefore, from (54), (55) and (57a)-(57d) one can write

$$
\begin{aligned}
\dot{\bar{V}} \leq & \bar{\xi}^{T}(t, \tau(t))\left(\bar{Z}_{i}+\bar{\Gamma}\right) \bar{\xi}(t, \tau(t)) \\
& +h^{-1} \int_{t-\tau(t)}^{t} \eta^{T}(t, s, \tau(s))\left(\bar{Y}_{j(s)}+\bar{\Omega}\right) \eta(t, s, \tau(s)) d s
\end{aligned}
$$

Similar to the proof of Theorem 1, note that inequality (34) implies

$$
\bar{\xi}^{T}(\cdot)\left(\bar{Z}_{i}+\bar{\Gamma}+\tilde{\bar{E}}_{i}^{T} \tilde{\bar{U}}_{i} \tilde{\bar{E}}_{i}\right) \xi(\cdot)<0
$$

using the Schur complement, where $\tilde{\bar{U}}_{i}$ and $\tilde{\bar{E}}_{i}$ are defined in Theorem 1. Note also that (28) and (34) imply $\bar{\xi}^{T}(\cdot)\left(\bar{Z}_{i}+\bar{\Gamma}\right) \bar{\xi}(\cdot)<0, x(t) \in X_{i}, i \in I_{1}$. Furthermore, (33) implies $\bar{Y}_{j(s)}+\bar{\Omega} \leq 0$ and from (58), $\dot{\bar{V}}_{i}<0$, $x(t) \in X_{i}, i \in I_{1}$. A similar procedure can be repeated for the case when the switching index belongs to $I_{0}$ leading to (30)-(32) and $\dot{V}_{i}<0, x(t) \in X_{i}, i \in I_{0}$. Thus the system is asymptotic stable following the argument of Theorem 1.

Remark 3: Extension of the results of Theorem 2 to PWA time-delay systems with the following dynamics

$$
\dot{x}(t)=\left(A_{i}+\Delta A_{i}\right) x(t)+\sum_{l=1}^{L}\left(A_{d i_{l}}+\Delta A_{d i_{l}}\right) x\left(t-\tau_{l}(t)\right)+\left(a_{i}+\Delta a_{i}\right)+\sum_{l=1}^{L}\left(b_{i l}+\Delta b_{i l}\right) 1\left(t-\tau_{l}(t)\right)
$$

where $0<\tau_{l} \leq h_{l}, \quad \dot{\tau}_{l}(t) \leq d_{l}<1, A_{d i_{l}} \in R^{n \times n}, \quad \tau_{l}(t) \in R^{+}, \quad$ and $l \in N$, is straightforward and is not developed in this paper due to space constraints. 


\section{$5 \quad$ Numerical Example}

In this section, four examples are provided to show the effectiveness of the proposed approach.

Example 1: In this example the stability of a time-delay system is investigated and it is shown that while the LMIs proposed in Kulkarni et al. (2004) are infeasible, the ones introduced in this paper are quite effective. Consider the piecewise linear time-delay system $\dot{x}(t)=A_{i} x(t)+A_{d i} x(t-\tau)$ with the system matrices given by

$$
\begin{gathered}
A_{1}=A_{3}=-\left[\begin{array}{cc}
0.1 & 0 \\
0 & 0.1
\end{array}\right] \quad A_{2}=A_{4}=-\left[\begin{array}{cc}
0.3 & 0 \\
0 & 0.3
\end{array}\right] \\
A_{d 1}=A_{d 3}=\left[\begin{array}{ll}
0 & 0 \\
0 & 0
\end{array}\right] \quad A_{d 2}=A_{d 4}=\left[\begin{array}{ll}
5 & 0 \\
0 & 0
\end{array}\right]
\end{gathered}
$$

and let the cell partition be given by

$$
E_{1}=-E_{3}=\left[\begin{array}{cc}
-1 & 1 \\
-1 & -1
\end{array}\right] \quad E_{2}=-E_{4}=\left[\begin{array}{cc}
-1 & 1 \\
1 & 1
\end{array}\right]
$$

One can verify that using the LMIs proposed in Kulkarni et al. (2004), stability of the system is guaranteed only for time-delays less than 0.005 , which is a very small margin. However, the LMIs derived in Theorem 1 ensure the stability for the time-delays as large as $h=10^{5}$.

Example 2: Consider the piecewise linear time-delay system $\dot{x}(t)=A_{i} x(t)+A_{d i} x(t-\tau)$, with the same cell partition as in (60), and the system matrices given by

$$
\begin{gathered}
A_{1}=A_{3}=-\left[\begin{array}{ll}
1 & 0 \\
0 & 1
\end{array}\right], \quad A_{2}=A_{4}=-\left[\begin{array}{cc}
0.9 & 0 \\
0 & 0.9
\end{array}\right] \\
A_{d 1}=A_{d 3}=\left[\begin{array}{cc}
0.1 & 5.0 \\
-5.0 & 0.1
\end{array}\right], \quad A_{d 2}=A_{d 4}=\left[\begin{array}{cc}
1.0 & 5.0 \\
-5.0 & -1.0
\end{array}\right]
\end{gathered}
$$

The LMIs derived in Theorem 1 are feasible for time-delays less than or equal to $h=0.0264$ in this example. Using simulation the system is unstable for $\tau_{\max }=0.031$. This seems to indicate that the result obtained in this example using the approach proposed for systems with no uncertainty is not too conservative.

Assume now that the matrices $A_{i}$ and $A_{d i}(i=1, \ldots, 4)$ in the above example are subject to uncertainty. It can be verified that for $\left\|\Delta A_{i}\right\| \leq 0.1$ and $\left\|\Delta A_{d i}\right\| \leq 0.1(i=1, \ldots, 4)$ the LMIs given in Theorem 2 are feasible for the time-delays less than or equal to $h=0.024$.

Example 3: In Fig. 1 a water tank and a pipe with the length of $L(m)$ are shown. In this example a nonlinear model of a water tank is considered as follows (Franklin et al. 2002)

$$
\dot{x}(t)=\frac{1}{A \rho}\left(u_{i n}(t-\tau(t))-\frac{1}{R} \sqrt{\rho g x(t)}\right)
$$

where $\rho=1000 \frac{K g}{m^{3}}, g=9.8 \frac{m}{s^{2}}, A=10 m^{2}$ and $R=11.3882 \frac{1}{\sqrt{m K g}}$. The output pipe diameter of the water tank is assumed to be $D=0.2 \mathrm{~m}$. It is assumed that the pipe length $L(\mathrm{~m})$ causes a delay $\tau$ for the input water to drop to the tank. It is desired to keep the level of water inside the tank at $x=0.5 \mathrm{~m}$. To this end, the PWA model of the above nonlinear system around two points, $x_{0}=0.25$ and $x_{0}=0.75$ is obtained 


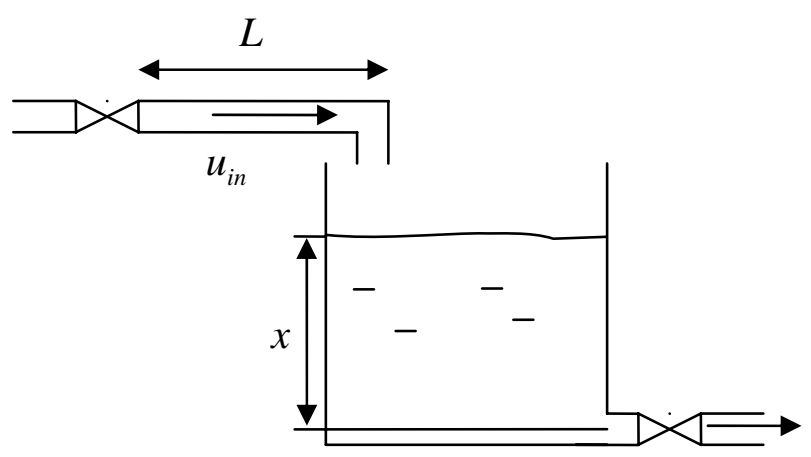

Figure 1. Water tank

$$
\begin{gathered}
\dot{x}(t)=-\frac{g x(t)}{2 A R \sqrt{0.25 \rho g}}+\left(\frac{0.25 g}{2 A R \sqrt{0.25 \rho g}}-\frac{\sqrt{0.25 \rho g}}{\rho A R}\right)+\frac{1}{A \rho} u_{i n}(t-\tau(t))+\Delta a_{1}, \quad 0 \leq x<0.5 \\
\dot{x}(t)=-\frac{g x(t)}{2 A R \sqrt{0.75 \rho g}}+\left(\frac{0.75 g}{2 A R \sqrt{0.75 \rho g}}-\frac{\sqrt{0.75 \rho g}}{\rho A R}\right)+\frac{1}{A \rho} u_{i n}(t-\tau(t))+\Delta A_{2} x(t), \quad 0.5 \leq x \leq 1
\end{gathered}
$$

where $\left\|\Delta a_{1}\right\| \leq 0.04$ and $\left\|\Delta A_{2}\right\| \leq 0.01$ represent the approximation error due to the linearization, and can be treated as uncertainties. Assume a control input of the form

$$
\begin{aligned}
& u_{\text {in }}(t)=A \rho[-0.111(t)-0.2(x(t)-0.51(t))], \quad 0 \leq x(t)<0.5 \\
& u_{\text {in }}(t)=-0.1 A \rho x(t), \quad 0.5 \leq x(t) \leq 1
\end{aligned}
$$

Using the LMIs derived in Theorem 2 the maximum delay is $h=1.57 \mathrm{~s}$. This implies that, e.g., by assuming the water speed be constant $u_{i n}=1 \frac{\mathrm{m}}{\mathrm{s}}$, the maximum pipe length must be less than $L=u_{i n} h=$ $1.57 \mathrm{~m}$.

Example 4: Consider the equation of motion of a simple pendulum (Franklin et al. 2002) as follows

$$
T(t-\tau(t))-m g l \sin (\theta(t))=m l^{2} \ddot{\theta}(t)
$$

where $l$ is the length of the pendulum, $g$ is the gravitational acceleration, $m$ is the pendulum mass and $T$ is the input torque. It is assumed that the communication link between the sensor and the controller has the delay of $\tau(t)$. It is desired to keep the pendulum at $\theta=0.3491 \mathrm{rad}=20 \mathrm{deg}$. To that end, the nonlinear model (64) is linearized around $\theta_{0}=0.7854 \mathrm{rad}$ and $\theta_{0}=0 \mathrm{rad}$ as follows

$$
\begin{gathered}
{\left[\begin{array}{c}
\dot{\theta}(t) \\
\ddot{\theta}(t)
\end{array}\right]=\left[\begin{array}{cc}
0 & 1 \\
-\frac{g}{l} & 0
\end{array}\right]\left[\begin{array}{l}
\theta(t) \\
\dot{\theta}(t)
\end{array}\right]+\left[\begin{array}{c}
0 \\
\frac{1}{m l^{2}} T(t-\tau(t))
\end{array}\right]+\Delta A_{1} x(t), \quad 0 \leq \theta(t)<0.3927} \\
{\left[\begin{array}{l}
\dot{\theta}(t) \\
\ddot{\theta}(t)
\end{array}\right]=\left[\begin{array}{cr}
0 & 1 \\
-0.7071 \frac{g}{l} & 0
\end{array}\right]\left[\begin{array}{c}
\theta(t) \\
\dot{\theta}(t)
\end{array}\right]+\left[\begin{array}{c}
0 \\
\frac{1}{m l^{2}} T(t-\tau(t))
\end{array}\right]+\left[\begin{array}{c}
0 \\
-1.2625 \frac{g}{l}
\end{array}\right]+\Delta A_{2} x(t), \quad 0.3927 \leq \theta(t) \leq 0.7854}
\end{gathered}
$$

where similar to the previous example, $\left\|\Delta A_{1}\right\| \leq 1.4$ and $\left\|\Delta A_{2}\right\| \leq 1.2$ represent the approximation error 
due to the linearization, and can be treated as uncertainties. The controller input to be used is as follows

$$
\begin{aligned}
& T(t)=\left[\begin{array}{ll}
-16 & -8
\end{array}\right]\left[\begin{array}{c}
\theta(t-\tau(t)) \\
\dot{\theta}(t-\tau(t))
\end{array}\right]+0.31501(t), \quad 0 \leq \theta(t)<0.3927 \\
& T(t)=\left[\begin{array}{ll}
13.1296-8 & -8\left[\begin{array}{c}
\theta(t-\tau(t)) \\
\dot{\theta}(t-\tau(t))
\end{array}\right]+6.07251(t), \quad 0.3927 \leq \theta(t) \leq 0.7854
\end{array}\right.
\end{aligned}
$$

Now it is desired to find the upper bound on delay $\tau(t)$, such that the system be stable. Using the LMIs in Theorem 2 the maximum value of delay $\tau(t)$ becomes $h=0.026 \mathrm{~s}$.

\section{Conclusions}

In this paper, robust stability of a class of piecewise affine systems with time-varying delay is considered. It is assumed that the system is subject to bounded uncertainty. It is also assumed that the time delay is unknown and time-varying, but an upper bound on the magnitude of the delay and its rate of variation exist. Sufficient conditions in the form of LMIs are derived for robust stability of the system. Numerical examples are provided to show the usefulness of the proposed approach. The following open problems can be considered for future work: (i) finding less conservative LMIs using different Lyapunov functionals; (ii) robust performance analysis of time-delay PWA systems; (iii) finding stability conditions for other types of uncertainties such as polytopic and polynomial, and (iv) stability analysis for neutral-type time-delay PWA systems.

\section{Acknowledgments}

The authors would like to acknowledge the financial support from Natural Sciences and Engineering Research Council (NSERC) and Fonds Quebecois de la Recherche sur la Nature et les Technologies (FQRNT) for funding this research.

\section{References}

A.G. Aghdam and E.J. Davison, "Pseudo-decentralized switching control", Automatica, 39(2), pp. 317-324, 2003.

A.G. Aghdam and E.J. Davison, "Decentralized switching control for hierarchical systems", Automatica, 43(6), pp. 1092-1100, 2007.

S. Azuma and J. Imura, "Optimal control of sampled-data piecewise-affine systems and its application to CPU processing control", in Proceedings of the 42th IEEE Conference on Decision Control, Maui, Hawaii, 2003, pp. 161-166.

A.G. Beccuti, G. Papafotiou and M. Morari, "Optimal control of the boost dc-dc converter", in Proceedings of the 44th IEEE Conference on Decision Control, Seville, Spain, 2005, pp. 4457-4462.

S. Bibian and H. Jin, "Time delay compensation of digital control for DC switch mode power supplies using prediction techniques", IEEE Transactions on Power Electronics, 15(5), pp. 835-842, 2000.

Q.W. C. Lin and T.H. Lee, "A less conservative robust stability test for linear uncertain time-delay systems", IEEE Transactions on Automatic Control, 51(1), pp. 87-91, 2006.

X. Da, C. Cheng and B. Tang, "A linear matrix inequality approach for robust control of systems with delayed states", European Journal of Operational Research, 124, pp. 332-341, 2000.

G.F. Franklin, J.D. Powell and A. Emami-Naeini, Feedback Control of Dynamic Systems, Upper Saddle River, New Jersey: Prentice Hall, 2002.

E. Fridman and U. Shaked, "Parameter dependent stability and stabilization of uncertain time-delay systems", IEEE Transactions on Automatic Control, 48(5), pp. 861-866, 2003.

Q.L. Han and K.Q. Gu, "On robust stability of time-delay systems with norm bounded uncertainty", IEEE Transactions on Automatic Control, 46(9), pp. 1426-1431, 2001. 
A. Hassibi and S.P. Boyd, "Quadratic stabilization and control of piecewise-linear systems", in Proceedings of American Control Conference, Philadelphia, Pennsylvani, 1998, pp. 3659-3664.

M. Johansson, Piecewise Linear Control Systems, Berlin, Heidelberg: Springer, 2003.

V. Kulkarni, M. Jun and J. Hespanha, "Piecewise quadratic Lyapunov functions for piecewise affine time-delay systems", in American Control Conference, Boston, Massachusetts, 2004, pp. 3885-3889.

A. Momeni and A.G. Aghdam, "An adaptive tracking problem for a family of retarded time-delay plants", Int. Journal of Adaptive Control and Signal Processing, 21, pp. 885-910, 2007.

S. Mondie and V. Kharitonov, "Exponential estimates for retarded time-delay systems: an LMI approach", IEEE Transactions on Automatic Control, 50(2), pp. 268-273, 2005.

M. Parlakci, "Robust stability of uncertain time-varying state-delayed systems", IEE Proc. on Control Theory and Applications, 153(4), pp. 469-477, 2006.

L. Rodrigues, "Stability analysis of piecewise-affine systems using controlled invariant sets", Systems Ef Control Letters, 53(2), pp. 157-169, 2004.

L. Rodrigues and E. Boukas, "Piecewise-linear $\mathcal{H}_{\infty}$ controller synthesis with applications to inventory control of switched production systems", Automatica, 42(8), pp. 1245-1254, 2006.

L. Rodrigues and S.P. Boyd, "Piecewise-affine state feedback for piecewise-affine slab systems using convex optimization", Systems $\&$ Control Letters, 54(9), pp. 835-583, 2005.

L. Rodrigues and J.P. How, "Observer-based control of piecewise-affine systems", International Journal of Control, 76(5), pp. 459-477, 2003.

L. Rodrigues and J. How, "Toward unified analysis and controller synthesis for a class of hybrid systems", Nonlinear Analysis, 65(12), pp. 2216-2235, 2006.

J.H. Su, "Further results on the robust stability of linear systems with a single time delay", Systems 63 Control Letters, 23(5), pp. 375-379, 1994.

X.M. Sun, J. Zhao and D. Hill, "Stability and $L_{2}$-gain analysis for switched delay systems: a delay-dependent method", Automatica, 42(10), pp. 1769-1774, 2006a.

Y.G. Sun, L. Wong and G. Xie, "Stability of switched systems with time-varying delays: delay-dependent common Lyapunov functional approach", in Proceedings of American Control Conference, Minneapolis, Minnesota, 2006b, pp. 1544-1549.

M.M. Tousi, I. Karuei, S.H. Zad and A.G. Aghdam, "Supervisory control of switching control systems", Systems E Control Letters, 57, pp. 132-141, 2008.

E.I. Verriest, M.K.H. Fan and J. Kullstam, "Frequency domain robust stability criteria for linear delay systems", in Proceedings of the 32nd IEEE Conference on Decision and Control, San Antonio, Texas, 1993, pp. 3473-3478.

S.S. Wang, B.S. Chen and T.P. Lin, "Robust stability of uncertain time-delay systems", Int. Journal of Control, 46, pp. 963-976, 1987.

Y. Wang, L. Xie and de C.E. Souza, "Robust control of a class of uncertain nonlinear systems", Systems 83 Control Letters, 19, pp. 139-149, 1992.

Y. Wei, L. Rodrigues and B. Gordon, "Piecewise-affine control of a three DOF aerobatic helicopter", in Proceedings of American Control Conference, Minneapolis, Minnesota, 2006, pp. 3924-3929.

G.S. Zhai, Y. Sun, X.K. Chen and N.M. Anthony, "Stability and $L_{2}$ gain analysis for switched symmetric systems with time delay", in Proceedings of American Control Conference, Denver, Colorado, 2003, pp. $2682-2687$.

W. Zhang, M. Branicky, and S.M. Phillips, "Stability of networked control systems", IEEE Control Systems Magazine, 21(1), pp. 84-99, 2001. 